\title{
Study of Intelligent Diagnosis System for Mechanism Wear Fault Based on Fuzzy-Neural Networks
}

\author{
Sanmao Xie* \\ School of Mechanical Engineering, East China Jiaotong university, \\ Nanchang 330013, China \\ Tel.: +86-791-7046135; Fax: +86-791-7046122 \\ xiesanmao@ecjtu.jx.cn
}

\begin{abstract}
According to fuzziness and complexity of mechanism wear fault, a kind of intelligent diagnosis system for mechanism wear fault based on fuzzyneural network is put forward. The structure and learning method of fuzzy-neural network are introduced. This paper analysises how to create the characteristic vector of wear particles and standard wear particles spectrum by combine the characteristics of mechanism wear fault, and the fuzzy-neural network of mechanism wear fault intelligent diagnosis is built. Mechanism wear faults can be diagnosed to apply the fuzzy-neural network and fault causes are determined. This system is developed by applying MATLAB software and the GUI functione of MATLAB for the simplicity and intuition of diagnosis.
\end{abstract}

Keywords: wear, fault diagnosis, fuzzy-neural networks, study.

\section{Introduction}

In mechanical faults, the wear faults occupy a large proportion of faults. For this type of fault diagnosis, common method is to use the oil sample analysis. However, oil sample analysis often can'nt obtained complete wear particles information, we required abundant experience to identify wear particles and wear. Therefore, the study on computer recognition of wear particle, that is intelligent diagnosis, has important significance. Common identification theory and diagnostic techniques of intelligent diagnosis have gray theory, fuzzy diagnosis, neural network diagnostic technology and so on[1]. Fuzzy-neural network is combined with fuzzy theory and neural networks. It not only can describe the problem with fuzzy concepts, but also has strong learning ability and processing capabilities of data. According to fuzziness and complexity of mechanism wear fault, this paper analysises how to create the characteristic vector of wear particles and standard wear particles spectrum by combine the characteristics of mechanism wear fault, a fuzzy-neural network is introduced and an intelligent particale recognition system is attained.

\section{Structure of Fuzzy-Neural Network}

In general, the combination of fuzzy systems and neural networks has four modes: loose combination, parallel combination, Series combination and learning combination.

\footnotetext{
* Corresponding author.
} 
The Series combination is simple and easy to be achieved, so in this paper, Series combination is used.

According to fuzzy logic and neural network features, fuzzy-neural network is built as Figure1. It is composed of three modules[3]:

(1) Input fuzzy module: to complete the transformation form fault symptoms to input vector.

(2) ANN inference module: to apply BP neural network to complete the reasoning diagnosis process.

(3) Output clarity module: to complete the process from the output of BP neural network to the diagnosis result.

In fuzzy-neural network, first, the fault symptoms are quantified based on fuzzy theory, the input vector is attained, second, output vector is attained by the learning and reasoning of BP neural network, finally the results of fault diagnosis is attained by comparing output datda with each other.

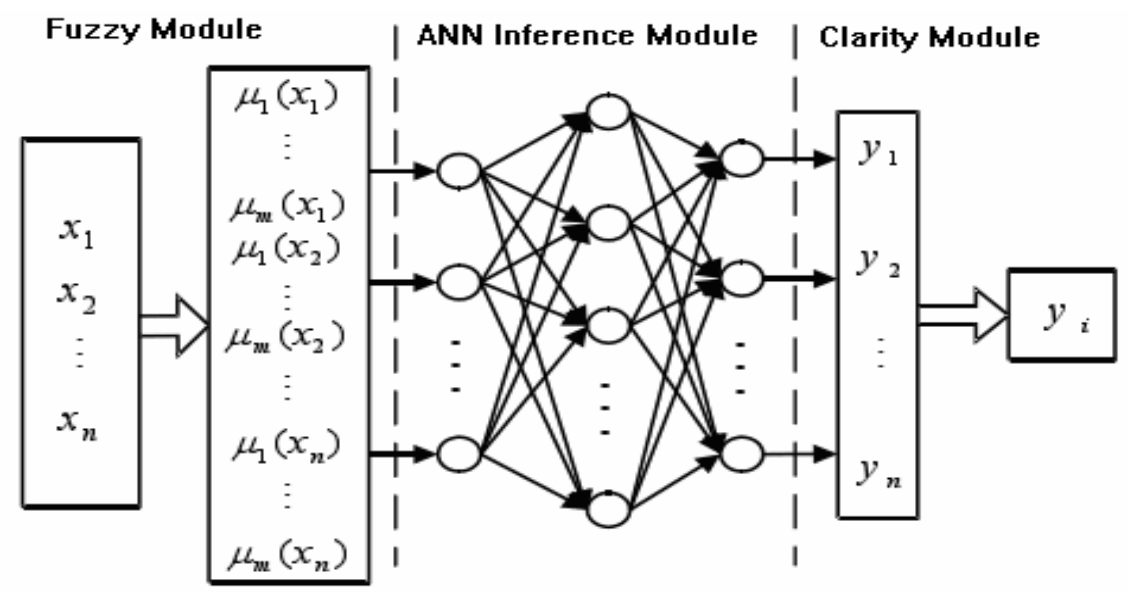

Fig. 1. Structure chart of fuzzy-neural network

\section{Establishment of Standard Particale Spectrum}

The priori knowledge of typical wear particls must be determined to recognize wear particles and classify wear, that is to establish standard particle spectrum. According to research, wear usually are divided into five basic types: normality, fatigue, abrasive, cutting and sliding. Relating to wear, wear particles are classified into 'normality', 'expanse slice', 'stick', 'sphere', 'cutting' and 'severe sliding'. Wear particle of normality is 'normality'. Wear particle of fatigue is 'expanse slice'or'stick'. Wear particle of abrasive is 'sphere'. Wear particles of cutting is 'cutting'. Wear particle of sliding is 'severe sliding'. This six kinds of particles can be described with the feature vector of five parameters such as outline shape, edge detail, surface texture, size, ratio of length to thickness[2]. As follows: 
$\mathrm{K}_{1}$ - outline shape, $\mathrm{K}_{2}$ - edge details, $\mathrm{K}_{3}$ - surface texture, $\mathrm{K}_{4}$ - size, $\mathrm{K}_{5}$-ratio of long-thickness

Based on the reference [1], [2] and linear principles, the following quantization values of particle features are obtained by combination of the particle characteristics and the typical morphology[1][2]:

outline shape: $\mathrm{K}_{11^{-}}$spherical shape(1.0), $\mathrm{K}_{12}$ - flake shape (0.8), $\mathrm{K}_{13}-$ circular shape (0.6), $\mathrm{K}_{14^{-}}$elongated shape (0.4).

edge details: $\mathrm{K}_{21^{-}}$smooth edge(1.0), $\mathrm{K}_{22^{-}}$straight edge $(0.8), \mathrm{K}_{23^{-}}$irregular edge(0.6)

surface texture: $\mathrm{K}_{31^{-}}$smooth surface (1.0), $\mathrm{K}_{32^{-}}$rough surface (0.8), $\mathrm{K}_{33^{-}}$striated surface (0.6), $\mathrm{K}_{34}$ - holed surface (0.4), $\mathrm{K}_{35^{-}}$pitted surface $(0.2)$

size: $\mathrm{K}_{41}<15 \mu \mathrm{m}(1.0), \mathrm{K}_{42}: 15 \sim 25 \mu \mathrm{m}(0.8), \mathrm{K}_{43}: 25 \sim 100 \mu \mathrm{m}(0.6), \mathrm{K}_{44}>100 \mu \mathrm{m}$ (0.4)

ratio of length to thickness: $\mathrm{K}_{51}<5: 1(1.0), \mathrm{K}_{52}: 5: 1 \sim 10: 1(0.8), \mathrm{K}_{53}>10: 1(0.6)$

The data in brackets express the quantization values of particle features. According to expert experience and knowledge, the following typical particles feature vectors are obtained:

'normality'particle feature vector:

$\mathrm{X}_{1}=(0.8,1.0,1.0,1.0,0.8)$

'expanse slice 'particle feature vector :

$\mathrm{X}_{2}=(0.8,0.6,0.4,0.6,0.6)$

'stick' particle feature vector :

$\mathrm{X}_{3}=(0.6,0.6,0.8,0.6,1.0)$

'sphere'particle feature vector:

$\mathrm{X}_{4}=(1.0,1.0,1.0,0.8,1.0)$

'cutting'particle feature vector:

$\mathrm{X}_{5}=(0.6,0.6,0.8,0.6,0.6)$

'severe sliding' particle feature vector:

$\mathrm{X}_{6}=(0.4,0.6,0.6,0.4,1.0)$

Thus the quantified standard particle spectrum is shown in table 1:

Table 1. Standard particle spectrum

\begin{tabular}{llllll}
\hline $\mathrm{X}_{\mathrm{i}}$ & \multicolumn{5}{c}{$\mathrm{K}_{\mathrm{m}}$} \\
\cline { 2 - 6 } & $\mathrm{K}_{1}$ & $\mathrm{~K}_{2}$ & $\mathrm{~K}_{3}$ & $\mathrm{~K}_{4}$ & $\mathrm{~K}_{5}$ \\
\hline $\mathrm{X}_{1}$ & 0.8 & 1.0 & 1.0 & 1.0 & 0.8 \\
$\mathrm{X}_{2}$ & 0.8 & 0.6 & 0.4 & 0.6 & 0.6 \\
$\mathrm{X}_{3}$ & 0.6 & 0.6 & 0.8 & 0.6 & 1.0 \\
$\mathrm{X}_{4}$ & 1.0 & 1.0 & 1.0 & 0.8 & 1.0 \\
$\mathrm{X}_{5}$ & 0.6 & 0.6 & 0.8 & 0.6 & 0.6 \\
$\mathrm{X}_{6}$ & 0.4 & 0.6 & 0.6 & 0.4 & 1.0 \\
\hline
\end{tabular}




\section{Procedure of Wear Fault Diagnosis Based on Fuzzy-Neural Network}

Procedure of wear fault diagnosis based on fuzzy-neural network is shown in figure 2 . Wear particle characteristic information are obtained from the oil sample analysis. After fuzzy processing, the feature vector of particles is provided to the diagnosis system by man-machine interaction. The diagnosis result of wear fault is shown by learning and reasoning of neural network.

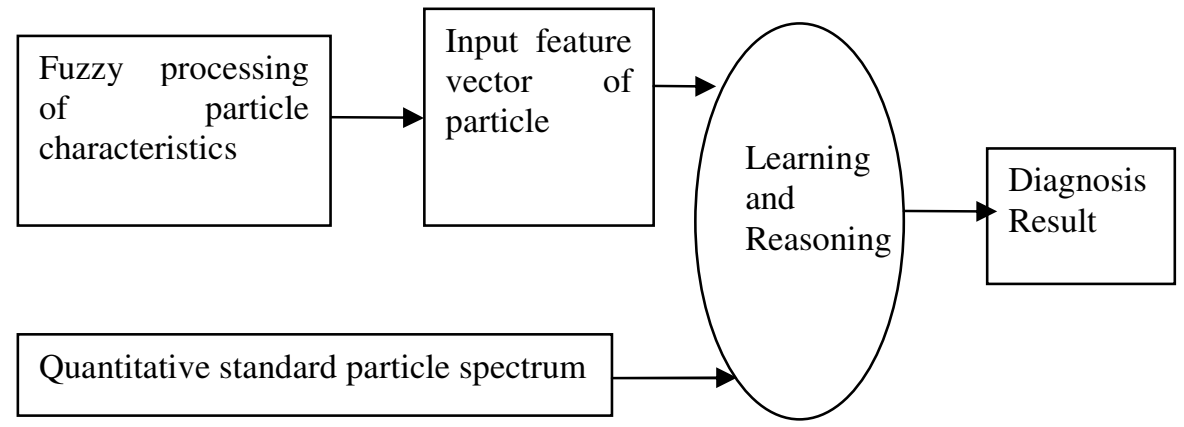

Fig. 2. Flow chart of wear fault diagnosis

\section{Design of ANN Inference Module}

\subsection{Structure of ANN Inference Module}

In this module, a three-layer BP neural network with input-layer, hidden-layer and output-layer is used and hybrid algorithm of 'BP-random' is adopted to train the network. Parameters of BP neural network are selected as following: (1) the number of input-layer node: As the five particle morphology parameters are as input after fuzzy, so 5 nodes are selected in input-layer; (2) the number of outputlayer node: Since there are six kinds of wear particle, so 6 nodes are selected in output-layer; (3) the number of hidden-layer node: According to the reference [1], the following formula can be used to determine the number of hidden-layer node: $l=\sqrt{n+m}+a, \mathrm{n}$ is the number of input-layer node, $\mathrm{m}$ is the number of outputlayer node, $\alpha$ is a constant between 1 to 10 . As a result, 10 nodes are selected in hidden-layer; (4) extraction of network training sample: According to the expert experience and knowledge, features spectrum of the typical wear particles has been shown in Table 1, so the features spectrum is used as the input training sample $\mathrm{P}$ of neural network. Table 2 shows the target output training samples $\mathrm{T}$ of the neural network, its output value is in the range $[0,1]$, the greater the value, the greater the likelihood of that type of fault. 
Table 2. The output matrix of the training sample

\begin{tabular}{ccccccc}
\hline & \multicolumn{7}{c}{ Output node } \\
\cline { 2 - 6 } Particles type & Node 1 & Node 2 & Node 3 & Node 4 & Node 5 & Node 6 \\
\hline 'normality' & 1 & 0 & 0 & 0 & 0 & 0 \\
'expanse slice' & 0 & 1 & 0 & 0 & 0 & 0 \\
'stick' & 0 & 0 & 1 & 0 & 0 & 0 \\
'sphere' & 0 & 0 & 0 & 1 & 0 & 0 \\
'cutting' & 0 & 0 & 0 & 0 & 1 & 0 \\
'severe sliding' & 0 & 0 & 0 & 0 & 0 & 1 \\
\hline
\end{tabular}

\subsection{Implementation of BP Neural Network}

BP neural network's basic idea is that characterized vector of training sample is as input of neural network, an output is get after the forward propagation calculation of neural network, and then the output is compared with a expected sample output, if there is deviation, the deviation is propagated back from the output layer, the network connection weights and thresholds are adjusted, the network's desired output is consistent to the output of the sample[4].

MATLAB software has stronge function of mathematical calculation, image processing and computer simulation and has a very high programming efficiency. It is more important that MATLAB concentrated the wisdom of experts and scholars in many fields and successfully expanded the toolbox of more than 30 fields, in which neural network toolbox is based on the theory of neural network. Utility functions of typical neural network is constructed in MATLAB. It provides a powerful and convenience tool for the application of the BP neural network[5][6].Therefore, this system is developed by applying MATLAB software and the GUI functione of MATLAB for the simplicity and intuition of diagnosis. BP neural network is created as follows:

net $=$ newff (minmax (P), [5 10 6], ('pureline"logsig' 'logsig'), 'trainlm')

Choose $\eta=0.2, \alpha=0.85$, and the system error is 0.001 , the corresponding network parameters:

net.trainParam. $\mathrm{mc}=0.85$

net.trainParam.lr = 0.2;

net.trainParam.goal $=0.001$;

The neural network is initialized and trained according to the samples of Table 1 and Table 2. As following:

net $=$ train $($ net, $P, T)$.

\section{The Main Function of This Intelligent Diagnosis System}

As MATLAB software has a powerful function of mathematical calculation and friendship GUI visual interface, so this system is developed by applying MATLAB software, the functions of system include: 
1) the system description;

2) neural network training;

3) wear particle recognition and wear fault diagnosis;

4) the help of system.

\section{Testing example of diagnostic system:}

According to the analysis of a particular ferrography, we obtained the characteristics information of wear particle tested. Feature vector $X_{0}$ was attained through fuzzy processing, as follows:

$$
\mathrm{X}_{0}=[0.6,1.0,0.8,0.6,0.8]
$$

In fact, these data is the wear particle membership of characteristics. Feature vector $\mathrm{X}_{0}$ was as the input of diagnosis system, the network output value is:

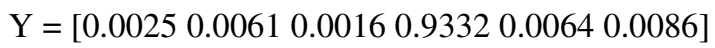

Obviously, the fourth is largest data of output nodes, the particle is 'sphere', the corresponding wear is abrasive wear. The diagnosis result is shown as Figure 3.

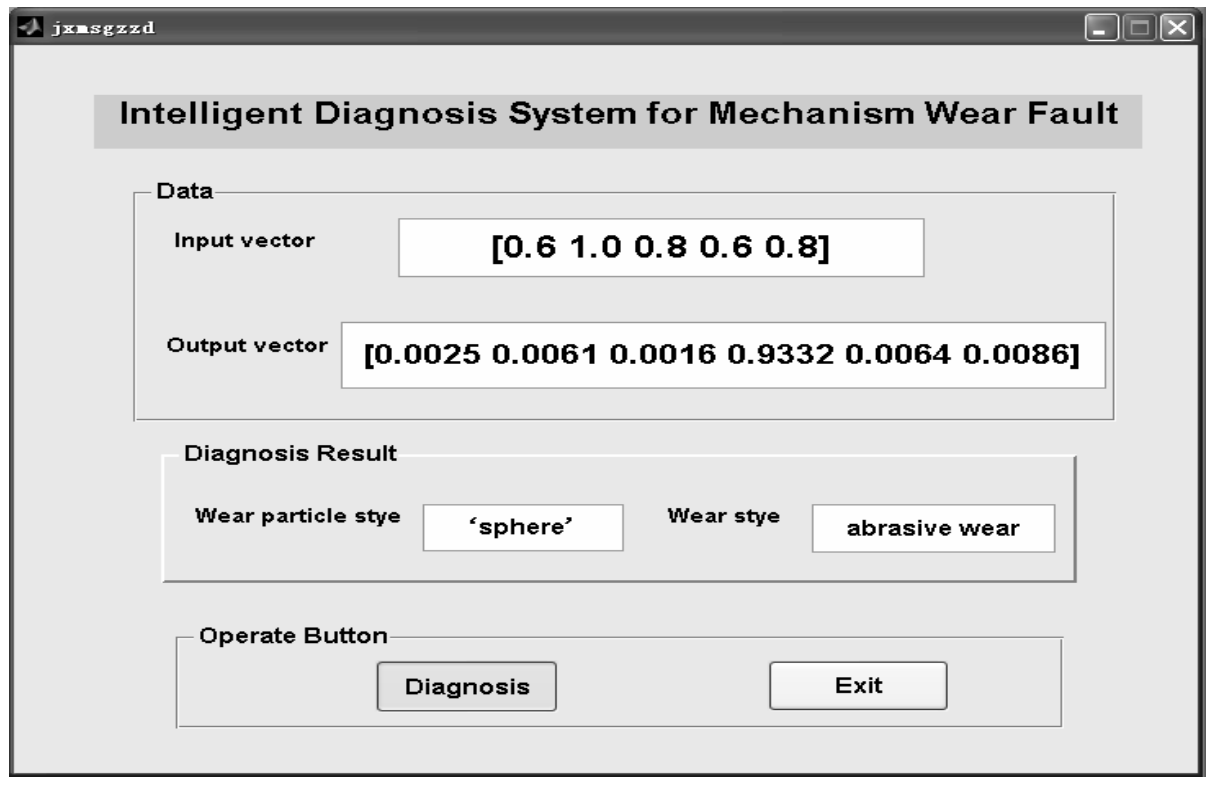

Fig. 3. Diagnosis result of wear fault

\section{Conclusion}

In this paper, mechanical wear faults were diagnosed effectively after obtaining characteristics information of wear particles and processing them with fuzzy theory. The effective combination of fuzzy theory and neural networks can handle ambiguous and even erroneous information. 


\section{References}

[1] Yu, H., Cheng, C.z., Zhang, s., Zhou, J.n.: Intelligent Diagnosis Based on Neural Networks. Metallurgy industry publishing house, Beijing (2002)

[2] Huang, A., Chen, Z.n., Zhu, J.m.: The Application of Artificial Neural Networks to Wear Debris Recognition in Ferrography Technology. Drive System Technique (1), 42-46 (1997)

[3] Li, G.-y., Yang, Q.-f.: FNN-based Intelligent Fault Diagnosis System for Vehicle Engine. Journal of System Simulation 19(5), 1033-1037 (2007)

[4] Li, G., Zhang, Y.: Machinery fault diagnosis. Chemistry industry publishing house, Beijing (1999)

[5] Zheng, A., Chao, y., Zhao, Y.: MATLAB Practical course. Electronic industry publishing house, Beijing (2004)

[6] Cong, S.: Neural Network Theory and Applications Based on MATLAB Toolbox. China Science and Technology University publishing house, Hefei (1998) 